\title{
Preconditioning Risk Factors Affecting Survival and Remission of Adult Egyptian Patients with Hematological Malignancies Undergoing Hematopoietic Stem Cell Transplantation
}

\author{
Fathy Ghamry Abd El-Razek, Ezzat Abd El-Rahman El-Etraby, Essam Abd El-Wahed Hassan, \\ Amro Mohamed Sedky El Ghammaz, Mohammed Abd El-Akher Mohammed * \\ Department of Internal Medicine, Faculty of Medicine, Al-Azhar University \\ *Correspondence author: Mohammed Abd El-Akher Mohammed, Mobile: (+20) (+20)01119998995
}

\begin{abstract}
Background: Hematopoietic stem cell transplantation (HSCT) was used for many years to treat various malignant and non-malignant hematologic conditions and in the treatment of various solid tumors. Since the 1970s, steady progress has been made and HSCT is now regarded as a routine, rather than an experimental, approach in the treatment of a number of conditions, which would have proven fatal earlier on.

Objective: Our study aimed to determine the most important preconditioning prognostic factors affecting the overall survival (OS), disease free survival (DFS) and relapse rate (RR) of adult Egyptians with hematological neoplasms who are treated with HSCT, whether autologous or allogeneic, and in turn minimizing the morbidity and mortality of those patients and improving their quality of life. Patients and methods: The study evaluated 98 adult patients with different hematological malignancies who underwent HSCT (whether autologous or allogeneic) at Stem Cell Transplantation Unit of Ain-Shams University from January 2014 to December 2018.

Results: In our study, in the group of allogenic transplantation, There was a statistically significance at overall survival with number of chemotherapy cycles with $\mathrm{P}=0.028$. Regarding post HSCT relapse and post HSCT mortality 11 patients (37.9\%) died in the group that received $\leq 4$ cycles of chemotherapy and 16 patients $(55.2 \%)$ died in the group that received $>4$ cycles of chemotherapy. Conclusion: Five-year overall and progression-free survival rose for allogeneic and autologous HSCT after the decade of their introduction.
\end{abstract}

Keywords: Hematological Malignancies, Hematopoietic Stem Cell Transplantation.

\section{INTRODUCTION}

Hematopoietic stem cell transplantation (HSCT) was used for many years to treat various malignant and non-malignant hematologic conditions and in the treatment of various solid tumors. Since the 1970s, steady progress was made and HSCT is now regarded as a routine, rather than an experimental, approach in the treatment of a number of conditions which would have proven fatal earlier on ${ }^{(1)}$.

Although stem cells can be collected by direct aspiration from the bone marrow, with the patient under general or spinal anesthetic, they are more commonly harvested from the peripheral blood. HSCT can be performed with cells from a family member or an unrelated volunteer (allogeneic transplantation), with stem cells previously collected from the patient (autologous transplantation) or with cells harvested from umbilical cord blood at time of delivery ${ }^{(2)}$.

It is well established that the cure of malignant disease by HSCT relies on both the conditioning regimen, which can provide an antitumor effect from myeloablative doses of chemotherapy or radiotherapy, and the graft-versus-leukemia $(\mathrm{GvL})$ effect provided by donor T-cells and NK cells. Malignant diseases have been found to have different susceptibilities to eradication by GvL effects, with decreasing susceptibility in diseases with rapid proliferation rates and advanced or chemorefractory disease ${ }^{(3)}$.

HSCT, particularly allogeneic transplantation, is a high cost and highly specialized procedure, performed by skilled and experienced transplant teams working in specialized centre. Allogeneic HSCT carries a relatively high mortality and morbidity. However, advances in supportive care and modifications made to the conditioning regimens played a central role in decreasing the morbidity and mortality of HSCT over the past years ${ }^{(4)}$.

\section{AIM OF THE WORK}

To determine the most important preconditioning prognostic factors affecting the overall survival (OS), disease free survival (DFS) and relapse rate (RR) of adult Egyptians with hematological neoplasms who are treated with HSCT whether autologous or allogeneic and in turn minimizing the morbidity and mortality of those patients and improving their quality of life.

\section{PATIENTS AND METHODS Patients:}

The study evaluated 98 adult patients with different hematological malignancies who underwent HSCT (whether autologous or allogeneic) at Stem Cell Transplantation Unit of Ain-Shams University from January 2014 to December 2018.

\section{Methods:}

GROUP I: Patients underwent autologous HSCT: peripheral blood mobilization of their stem cells was transfused to them after receiving conditioning. 
GROUP II: Patients underwent allogeneic HSCT received conditioning then transfusion of stem cells mobilized from the peripheral blood of a fully matched sibling donor, they received GVHD prophylaxis in the form of methotrexate and cyclosporine A.

\section{All patients were subjected to:}

- Detailed history taking

- Thorough physical examination

- $\mathrm{CBC}$ with differential

- ESR

- $\mathrm{ABO}$ group and Rhesus typing with antibody screen (allogeneic HSCT)

- Metabolic profile (kidney function tests, liver function tests, $\mathrm{LDH}$, uric acid)

- Creatinine clearance

- PT, INR, PTT

- CSF examination and cytospin (if indicated)

- BM aspiration and/or BM biopsy

- Initial cytogenetics

- Viral markers:

- HBsAg, HbeAg, anti-HBc (IgM/IgG), anti-HBs, anti$\mathrm{HBe}$ and PCR for HBV-DNA

- HCV-Ab, PCR for HCV-RNA

○ HIV-Ab

- CMV-IgM, CMV-IgG (allogeneic HSCT)

- EBV-IgM, EBV-IgG (allogeneic HSCT)

- HSV-IgM, HSV-IgG (allogeneic HSCT)

- Toxoplasma-IgM, Toxoplasma-IgG (allogenic HSCT)

- HLA typing (allogeneic HSCT)

- Chest X-ray

- CT scan of neck, chest and pelvi-abdomen (if indicated)

- MRI of brain, spine (if indicated)

- $\quad$ PET-CT (if indicated)

- Echocardiography

- Pulmonary function tests (allogeneic HSCT)

- Liver biopsy (if indicated)

- Dental check

Patients were followed up for a minimum period of 2 years and they were monitored for survival and remission states. Analysis of preconditioning risk factors were performed and correlated statistically to OS, DFS and RR.

\section{Written informed consent:}

An approval of the study was obtained from Al- Azhar University academic and ethical committee. Every patient signed an informed written consent for acceptance of the operation.

\section{Statistical Analysis}

Recorded data were analyzed using the statistical package for social sciences, version 20.0 (SPSS Inc., Chicago, Illinois, USA). Quantitative data were expressed as mean \pm standard deviation (SD). Qualitative data were expressed as frequency and percentage.

\section{The following tests were done:}

- Chi-square $\left(\mathbf{x}^{2}\right)$ test of significance was used in order to compare proportions between qualitative parameters.

- Kaplan-Meier Survival Analysis: is a descriptive procedure for examining the distribution of time-to-event variables.

- Log rank test to compare time-to-event variables by levels of a factor variable.

- The confidence interval was set to $95 \%$ and the margin of error accepted was set to 5\%. So, the pvalue was considered significant as the following:

- Probability (P-value)

- P-value $\leq 0.05$ was considered significant.

- P-value $\leq 0.001$ was considered as highly significant.

- $\quad$ P-value >0.05 was considered insignificant.

\section{RESULTS}

Demographic data: (Table 1)

Table (1): Demographic data distribution of the study group.

\begin{tabular}{|c|c|}
\hline Demographic Data & Total $(\mathbf{n = 9 8})$ \\
\hline Age $($ years) & \\
$>50$ years & $83(84.7 \%)$ \\
$\leq 50$ years & $15(15.3 \%)$ \\
\hline Range [Mean \pm SD] & $21-60[37.87 \pm 10.39]$ \\
\hline Sex & $58(59.2 \%)$ \\
Male & $40(40.8 \%)$ \\
Female & $69(70.4 \%)$ \\
Performance Status & $29(29.6 \%)$ \\
\hline 0 & \\
\hline
\end{tabular}

Group I (autologous HSCT) overall survival: There was a statistically significance of overall survival and post HSCT mortality with previous radiotherapy within the group with autologous HSCT with (p value was 0.002 ) as shown in tables 2. 
ejhm.journals.ekb.eg

Table (2): Overall survival between all parameters in group I: Autologous.

\begin{tabular}{|c|c|c|c|c|c|c|c|}
\hline \multirow{2}{*}{ Group I: Autologous (n=37) } & \multirow{2}{*}{$\begin{array}{c}\text { Total } \\
\text { mortality } \\
(n=11)\end{array}$} & \multirow{2}{*}{$\begin{array}{c}\text { Median } \\
\text { of OS }\end{array}$} & \multirow[b]{2}{*}{ SE } & \multicolumn{2}{|c|}{$95 \%$ C.I. } & \multirow{2}{*}{$\begin{array}{l}\text { Chi- } \\
\text { Square }\end{array}$} & \multirow[b]{2}{*}{$\mathbf{P}$} \\
\hline & & & & Lower & Upper & & \\
\hline \multicolumn{8}{|l|}{ Demographic data } \\
\hline \multicolumn{8}{|l|}{ Age (years) } \\
\hline$>50$ years & $5(45.5 \%)$ & 32.00 & 7.19 & 17.91 & 46.09 & \multirow{2}{*}{2.849} & \multirow{2}{*}{0.091} \\
\hline$\leq 50$ years & $6(54.5 \%)$ & 17.00 & 12.16 & 0.00 & 40.83 & & \\
\hline \multicolumn{8}{|l|}{ Sex } \\
\hline Male & $8(72.7 \%)$ & 24.00 & 3.82 & 16.50 & 31.50 & \multirow{2}{*}{0.119} & \multirow{2}{*}{0.730} \\
\hline Female & $3(27.3 \%)$ & 28.00 & 15.41 & 0.00 & 58.21 & & \\
\hline \multicolumn{8}{|l|}{ Performance Status } \\
\hline 0 & $5(45.5 \%)$ & 28.00 & 5.00 & 18.20 & 37.80 & \multirow{2}{*}{0.695} & \multirow{2}{*}{0.405} \\
\hline 1 & $6(54.5 \%)$ & 24.00 & 2.29 & 19.51 & 28.49 & & \\
\hline \multicolumn{8}{|l|}{ Co-morbidities } \\
\hline No & $6(54.5 \%)$ & 24.00 & 8.37 & 7.59 & 40.41 & \multirow{2}{*}{0.008} & \multirow{2}{*}{0.929} \\
\hline Yes & $5(45.5 \%)$ & 28.00 & 7.62 & 13.06 & 42.94 & & \\
\hline \multicolumn{8}{|l|}{ Disease of diagnosis } \\
\hline AML & $0(0.0 \%)$ & 22.50 & 5.50 & 11.72 & 33.28 & \multirow{4}{*}{5.098} & \multirow{4}{*}{0.165} \\
\hline ALL & $0(0.0 \%)$ & 73.00 & 0.00 & 73.00 & 73.00 & & \\
\hline Lymphoma & $4(36.4 \%)$ & 29.81 & 5.98 & 18.08 & 41.53 & & \\
\hline Multiple myeloma & $7(63.6 \%)$ & 26.43 & 3.82 & 18.95 & 33.91 & & \\
\hline \multicolumn{8}{|l|}{ No of Chemotherapy Cycles } \\
\hline$\leq 4$ cycles & $1(9.1 \%)$ & 40.00 & 6.98 & 26.33 & 53.67 & & \\
\hline$>4$ cycles & $\begin{array}{c}10 \\
(90.9 \%) \\
\end{array}$ & 27.29 & 3.48 & 20.47 & 34.10 & 0.634 & 0.426 \\
\hline Previous Radiotherapy & & & & & & & \\
\hline No & $5(45.5 \%)$ & 37.00 & 9.99 & 17.42 & 56.58 & 0.587 & ค $007 *$ \\
\hline Yes & $6(54.5 \%)$ & 10.00 & 7.51 & 0.00 & 24.71 & 9.581 & $0.002^{*}$ \\
\hline Disease Stage & & & & & & & \\
\hline Complete remission & $8(72.7 \%)$ & 28.00 & 6.16 & 15.92 & 40.08 & & \\
\hline Partial remission & $3(27.3 \%)$ & 16.00 & 5.24 & 5.74 & 26.26 & 0.764 & 0.382 \\
\hline $\begin{array}{l}\text { Time from diagnosis to HCT } \\
\text { (months) }\end{array}$ & & & & & & & \\
\hline$>6-12$ months & $5(45.5 \%)$ & 28.00 & 2.71 & 22.70 & 33.30 & & \\
\hline$>12-18$ months & $6(54.5 \%)$ & 17.00 & 12.30 & 0.00 & 41.10 & 0.546 & 0.460 \\
\hline CMV status & & & & & & & \\
\hline Positive & $0(0.0 \%)$ & 49.00 & 21.23 & 7.39 & 90.61 & & \\
\hline Negative & $\begin{array}{c}11 \\
(100.0 \%)\end{array}$ & 25.00 & 3.50 & 18.14 & 31.86 & 0.836 & 0.361 \\
\hline Cytogenetics & & & & & & & \\
\hline Unfavourable risk & $2(18.2 \%)$ & 33.08 & 5.32 & 22.65 & 43.52 & & \\
\hline Favourable risk & $0(0.0 \%)$ & 11.50 & 5.50 & 0.72 & 22.28 & 3.588 & 0.166 \\
\hline Not available & $9(81.8 \%)$ & 27.85 & 4.28 & 19.45 & 36.24 & & \\
\hline Post HSCT: Relapse & & & & & & & \\
\hline Positive & $6(54.5 \%)$ & 28.00 & 2.92 & 22.27 & 33.73 & 0070 & 0865 \\
\hline Negative & $5(45.5 \%)$ & 24.00 & 9.80 & 4.80 & 43.20 & 0.029 & 0.865 \\
\hline
\end{tabular}

Group I (autologous HSCT) Disease free survival: There was no statistically significance in disease free survival regarding all parameters with $\mathrm{p}$ value $>0.05$ as shown in tables 3 . 
Table (3): Disease free survival between all parameters in group I: Autologous.

\begin{tabular}{|c|c|c|c|c|c|c|c|}
\hline \multirow{2}{*}{$\begin{array}{l}\text { Group I: Autologous } \\
(\mathbf{n}=37)\end{array}$} & \multirow{2}{*}{$\begin{array}{l}\text { Total } \\
\text { relapse } \\
(\mathbf{n}=\mathbf{6})\end{array}$} & \multirow{2}{*}{$\begin{array}{l}\text { Median } \\
\text { of DFS }\end{array}$} & \multirow[b]{2}{*}{ SE } & \multicolumn{2}{|c|}{ 95\% C.I. } & \multirow{2}{*}{$\begin{array}{l}\text { Chi- } \\
\text { Square }\end{array}$} & \multirow{2}{*}{$\mathbf{P}$} \\
\hline & & & & Lower & Upper & & \\
\hline \multicolumn{8}{|l|}{ Demographic data } \\
\hline \multicolumn{8}{|l|}{ Age (years) } \\
\hline$>50$ years & $4(66.7 \%)$ & 22.00 & 8.94 & 4.47 & 39.53 & \multirow{2}{*}{0.948} & \multirow{2}{*}{0.330} \\
\hline$\leq 50$ years & $2(33.3 \%)$ & 18.00 & 6.00 & 6.24 & 29.76 & & \\
\hline \multicolumn{8}{|l|}{ Sex } \\
\hline Male & $5(83.3 \%)$ & 18.00 & 4.74 & 8.70 & 27.30 & \multirow{2}{*}{0.113} & \multirow{2}{*}{0.737} \\
\hline Female & $1(16.7 \%)$ & 22.00 & 6.53 & 9.20 & 34.80 & & \\
\hline \multicolumn{8}{|l|}{ Performance Status } \\
\hline 0 & $3(50.0 \%)$ & 14.00 & 6.74 & 0.80 & 27.20 & \multirow{2}{*}{0.293} & \multirow{2}{*}{0.589} \\
\hline 1 & $3(50.0 \%)$ & 22.00 & 5.24 & 11.74 & 32.26 & & \\
\hline \multicolumn{8}{|l|}{ Co-morbidities } \\
\hline No & $4(66.7 \%)$ & 18.00 & 9.17 & 0.04 & 35.96 & \multirow{2}{*}{0.538} & \multirow{2}{*}{0.463} \\
\hline Yes & $2(33.3 \%)$ & 22.00 & 4.29 & 13.60 & 30.40 & & \\
\hline \multicolumn{8}{|l|}{ Disease of diagnosis } \\
\hline AML & $0(0.0 \%)$ & 14.00 & 0.00 & 14.00 & 14.00 & \multirow{3}{*}{3.120} & \multirow{3}{*}{0.210} \\
\hline Lymphoma & $2(33.3 \%)$ & 26.25 & 8.50 & 9.59 & 42.91 & & \\
\hline Multiple myeloma & $4(66.7 \%)$ & 18.63 & 2.07 & 14.57 & 22.68 & & \\
\hline \multicolumn{8}{|l|}{$\begin{array}{l}\text { No of Chemotherapy } \\
\text { Cycles }\end{array}$} \\
\hline$\leq 4$ cycles & $1(16.7 \%)$ & 22.00 & 6.53 & 9.20 & 34.80 & \multirow{2}{*}{0.831} & \multirow{2}{*}{0.362} \\
\hline$>4$ cycles & $5(83.3 \%)$ & 18.00 & 4.74 & 8.70 & 27.30 & & \\
\hline \multicolumn{8}{|c|}{ Previous Radiotherapy } \\
\hline No & $3(50.0 \%)$ & 22.00 & 5.24 & 11.74 & 32.26 & & \\
\hline Yes & $3(50.0 \%)$ & 16.00 & 7.35 & 1.60 & 30.40 & 1.810 & 0.178 \\
\hline Disease Stage & & & & & & & \\
\hline Complete remission & $5(83.3 \%)$ & 22.00 & 3.10 & 15.93 & 28.07 & & \\
\hline Partial remission & $1(16.7 \%)$ & 16.00 & 4.08 & 8.00 & 24.00 & 1.075 & 0.300 \\
\hline $\begin{array}{l}\text { Time from diagnosis to } \\
\text { HCT (months) }\end{array}$ & & & & & & & \\
\hline$>6-12$ months & $3(50.0 \%)$ & 22.00 & 3.54 & 15.07 & 28.93 & 040 & \\
\hline$>12-18$ months & $3(50.0 \%)$ & 11.00 & 1.10 & 8.85 & 13.15 & 940 & 0.332 \\
\hline CMV status & & & & & & & \\
\hline Positive & $0(0.0 \%)$ & 28.00 & 5.00 & 18.20 & 37.80 & & \\
\hline Negative & $\begin{array}{c}6 \\
(100.0 \%) \\
\end{array}$ & 19.27 & 3.21 & 12.99 & 25.56 & 0.694 & 0.405 \\
\hline Cytogenetics & & & & & & & \\
\hline Unfavourable risk & $2(33.3 \%)$ & 22.00 & 4.90 & 12.40 & 31.60 & & \\
\hline Not available & $4(66.7 \%)$ & 18.00 & 9.17 & 0.04 & 35.96 & 1.189 & 0.275 \\
\hline
\end{tabular}

Group II (allogeneic HSCT) overall survival: There was a statistically significance at overall survival with performance status, disease of diagnosis, No of chemotherapy cycles with $\mathrm{P}$ value was $(0.027,0.018$ and 0.028 respectively) regarding post HSCT relapse and post HSCT mortality. 
ejhm.journals.ekb.eg

Table (4): Overall survival between all parameters in group II: allogeneic

\begin{tabular}{|c|c|c|c|c|c|c|c|}
\hline \multirow{2}{*}{$\begin{array}{l}\text { Group II: Allogenic } \\
(n=61)\end{array}$} & \multirow{2}{*}{$\begin{array}{c}\text { Total } \\
\text { mortality } \\
(\mathbf{n}=29)\end{array}$} & \multirow{2}{*}{$\begin{array}{l}\text { Median } \\
\text { of OS }\end{array}$} & \multirow[b]{2}{*}{ SE } & \multicolumn{2}{|c|}{ 95\% C.I. } & \multirow{2}{*}{$\begin{array}{l}\text { Chi- } \\
\text { Square }\end{array}$} & \multirow[b]{2}{*}{$\mathbf{P}$} \\
\hline & & & & Lower & Upper & & \\
\hline \multicolumn{8}{|l|}{\begin{tabular}{|l|} 
Demographic data \\
\end{tabular}} \\
\hline \multicolumn{8}{|l|}{ Age (years) } \\
\hline$>50$ years & $28(96.6 \%)$ & 33.69 & 4.13 & 25.59 & 41.79 & \multirow[b]{2}{*}{0.345} & \multirow{2}{*}{0.557} \\
\hline$\leq 50$ years & $1(3.4 \%)$ & 18.00 & 0.00 & 18.00 & 18.00 & & \\
\hline \multicolumn{8}{|l|}{ Sex } \\
\hline Male & $16(55.2 \%)$ & 20.00 & 4.53 & 11.13 & 28.87 & \multirow{2}{*}{0.734} & \multirow{2}{*}{0.392} \\
\hline Female & $13(44.8 \%)$ & 29.00 & 6.28 & 16.69 & 41.31 & & \\
\hline \multicolumn{8}{|l|}{\begin{tabular}{|l|} 
Performance Status \\
\end{tabular}} \\
\hline 0 & $25(86.2 \%)$ & 30.00 & 7.28 & 15.73 & 44.27 & \multirow{2}{*}{4.864} & \multirow{2}{*}{$0.027 *$} \\
\hline 1 & $5(17.2 \%)$ & 6.00 & 3.54 & 0.00 & 12.93 & & \\
\hline \multicolumn{8}{|l|}{\begin{tabular}{|l} 
Co-morbidities \\
\end{tabular}} \\
\hline No & $9(31.0 \%)$ & 37.00 & 5.39 & 26.44 & 47.56 & \multirow[b]{2}{*}{1.077} & \multirow{2}{*}{0.299} \\
\hline Yes & $20(69.0 \%)$ & 18.00 & 4.62 & \begin{tabular}{|l|}
8.95 \\
\end{tabular} & 27.05 & & \\
\hline \multicolumn{8}{|l|}{ Disease of diagnosis } \\
\hline AML & $15(51.7 \%)$ & 32.63 & 4.95 & 22.93 & 42.32 & \multirow{3}{*}{7.997} & \multirow{3}{*}{$0.018 *$} \\
\hline CML & $2(6.9 \%)$ & 68.17 & 21.22 & 26.57 & 109.76 & & \\
\hline ALL & $12(41.4 \%)$ & 24.00 & 4.64 & 14.90 & 33.10 & & \\
\hline \multicolumn{8}{|l|}{$\begin{array}{l}\text { No of Chemotherapy } \\
\text { Cycles }\end{array}$} \\
\hline No & $2(6.9 \%)$ & 68.17 & 21.22 & 26.57 & 109.76 & & \\
\hline$\leq 4$ cycles & $11(37.9 \%)$ & 33.57 & 5.53 & 22.74 & 44.40 & 7.166 & $0.028 *$ \\
\hline$>4$ cycles & $16(55.2 \%)$ & 25.27 & 4.54 & 16.38 & 34.16 & & \\
\hline Previous Radiother & & & & & & & \\
\hline No & $25(86.2 \%)$ & 35.26 & 4.40 & 26.64 & 43.89 & & \\
\hline Yes & $5(17.2 \%)$ & 16.67 & 6.46 & 4.00 & 29.34 & 2.701 & 0.100 \\
\hline $\begin{array}{l}\text { Donor/host Sex } \\
\text { Combination }\end{array}$ & & & & & & & \\
\hline Same sex & $25(86.2 \%)$ & 23.00 & 7.21 & 8.87 & 37.13 & & \\
\hline Different sex & $4(13.8 \%)$ & 21.00 & 4.47 & 12.23 & 29.77 & 0.040 & 0.841 \\
\hline $\begin{array}{l}\text { Time from diagnosi } \\
\text { HCT (months) }\end{array}$ & & & & & & & \\
\hline$\leq 6$ months & $1(3.4 \%)$ & 42.00 & 19.00 & 4.76 & 79.24 & & \\
\hline$>6-12$ months & $16(55.2 \%)$ & 23.00 & 9.12 & 5.12 & 40.88 & 1.443 & 0.486 \\
\hline$>12-18$ months & $12(41.4 \%)$ & 18.00 & 7.83 & 2.66 & 33.34 & & \\
\hline CMV status & & & & & & & \\
\hline$D+/ R+$ & $0(0.0 \%)$ & 37.00 & 0.00 & 37.00 & 37.00 & 0.004 & 0.952 \\
\hline$D+/ R-$ & $0(0.0 \%)$ & - & - & - & - & - & - \\
\hline$D-/ R+$ & $1(3.4 \%)$ & 42.00 & 0.00 & 42.00 & 42.00 & 0.016 & 0.899 \\
\hline$D-/ R-$ & $28(96.6 \%)$ & 33.23 & 4.21 & 24.98 & 41.48 & 0.003 & 0.957 \\
\hline Cytogenetics & & & & & & & \\
\hline Unfavourable risk & $25(86.2 \%)$ & 34.27 & 4.22 & 26.01 & 42.54 & & \\
\hline Favourable risk & $3(10.3 \%)$ & 11.13 & 2.55 & 6.12 & 16.13 & 3.687 & 0.158 \\
\hline Not available & $1(3.4 \%)$ & 55.00 & 49.00 & 0.00 & 151.04 & & \\
\hline
\end{tabular}


Table (5): Disease free survival between all parameters in group II: allogeneic.

\begin{tabular}{|c|c|c|c|c|c|c|c|}
\hline \multirow{2}{*}{ roup II: Allogenic $(n=61)$} & \multirow{2}{*}{$\begin{array}{c}\text { otal relapse } \\
(\mathrm{n}=6)\end{array}$} & \multirow{2}{*}{$\begin{array}{l}\text { Median } \\
\text { of DFS }\end{array}$} & \multirow{2}{*}{$\mathbf{S E}$} & \multicolumn{2}{|c|}{ 95\% C.I. } & \multirow{2}{*}{$\begin{array}{l}\text { Chi- } \\
\text { pquare }\end{array}$} & \multirow{2}{*}{$\mathbf{P}$} \\
\hline & & & & Lower & Upper & & \\
\hline \multicolumn{8}{|l|}{\begin{tabular}{|l|} 
Demographic data \\
\end{tabular}} \\
\hline \multicolumn{8}{|l|}{\begin{tabular}{|l|} 
Age (years) \\
\end{tabular}} \\
\hline$>50$ years & $5(83.3 \%)$ & 11.67 & 4.71 & 2.44 & 20.89 & \multirow{2}{*}{0.219} & \multirow{2}{*}{0.640} \\
\hline$\leq 50$ years & $1(16.7 \%)$ & 17.00 & 0.00 & 17.00 & 17.00 & & \\
\hline \multicolumn{8}{|l|}{ Sex } \\
\hline Male & $2(33.3 \%)$ & 6.00 & 0.82 & 4.40 & 7.60 & \multirow{2}{*}{0.211} & \multirow{2}{*}{0.646} \\
\hline Female & $4(66.7 \%)$ & 5.50 & 6.75 & 0.00 & 18.73 & & \\
\hline \multicolumn{8}{|l|}{ Performance Status } \\
\hline 0 & $6(100.0 \%)$ & 6.00 & 0.65 & 4.72 & 7.28 & \multirow[b]{2}{*}{ - } & \multirow[b]{2}{*}{ - } \\
\hline 1 & $0(0.0 \%)$ & - & - & - & - & & \\
\hline \multicolumn{8}{|l|}{ Co-morbidities } \\
\hline No & $3(50.0 \%)$ & 6.00 & 2.04 & 2.00 & 10.00 & \multirow{2}{*}{0.092} & \multirow{2}{*}{0.762} \\
\hline Yes & $3(50.0 \%)$ & 5.50 & 4.00 & 0.00 & 13.34 & & \\
\hline \multicolumn{8}{|l|}{ Disease of diagnosis } \\
\hline AML & $4(66.7 \%)$ & 9.70 & 3.01 & 3.81 & 15.59 & \multirow{2}{*}{0.814} & \multirow{2}{*}{0.367} \\
\hline ALL & $2(33.3 \%)$ & 19.25 & 13.75 & 0.00 & 46.20 & & \\
\hline \multicolumn{8}{|c|}{ No of Chemotherapy Cycles } \\
\hline$\leq 4$ cycles & $2(33.3 \%)$ & 14.67 & 9.17 & 0.00 & 32.64 & \multirow{2}{*}{0.203} & 0650 \\
\hline$>4$ cycles & $4(66.7 \%)$ & 10.75 & 3.63 & 3.63 & 17.87 & & 0.652 \\
\hline Previous Radiotherapy & & & & & & & \\
\hline No & $6(100.0 \%)$ & 6.00 & 0.65 & 4.72 & 7.28 & & \\
\hline Yes & $0(0.0 \%)$ & - & - & - & - & - & - \\
\hline $\begin{array}{l}\text { Donor/host Sex } \\
\text { Combination }\end{array}$ & & & & & & & \\
\hline Same sex & $6(100.0 \%)$ & 13.67 & 4.56 & 4.72 & 22.61 & 1824 & 0177 \\
\hline Different sex & $0(0.0 \%)$ & 5.00 & 0.00 & 5.00 & 5.00 & 1.824 & $0.17 /$ \\
\hline Time from diagnosis to $\mathrm{I}$ & CT (months) & & & & & & \\
\hline$\leq 6$ months & $1(16.7 \%)$ & 33.00 & 0.00 & 33.00 & 33.00 & & \\
\hline$>6-12$ months & $3(50.0 \%)$ & 5.00 & 0.54 & 3.94 & 6.06 & 6.239 & $0.044 *$ \\
\hline$>12-18$ months & $2(33.3 \%)$ & 17.00 & 0.00 & 17.00 & 17.00 & & \\
\hline CMV status & & & & & & & \\
\hline$D+/ R+$ & $0(0.0 \%)$ & - & - & - & - & - & - \\
\hline$D+/ R-$ & $0(0.0 \%)$ & - & - & - & - & - & - \\
\hline$D-/ R+$ & $1(16.7 \%)$ & 33.00 & 0.00 & 33.00 & 33.00 & 2.447 & 0.118 \\
\hline$D-/ R-$ & $5(83.3 \%)$ & 5.50 & 0.61 & 4.30 & 6.70 & 2.471 & 0.112 \\
\hline Cytogenetics & & & & & & & \\
\hline Unfavourable risk & $3(50.0 \%)$ & 14.63 & 6.83 & 1.24 & 28.01 & & \\
\hline Favourable risk & $2(33.3 \%)$ & 11.50 & 5.50 & 0.72 & 22.28 & 0.571 & 0.752 \\
\hline Not available & $1(16.7 \%)$ & 5.50 & 0.00 & 5.50 & 5.50 & & \\
\hline
\end{tabular}

This table shows statistically significant $\mathrm{P}$ Value of disease free survival with time from diagnosis to HCT months with $P$ value about 0.044 between patients of that time was $\leq 6$ months vs $>6-12$ months vs $>12-18$ months.

Table (6): Multivariate analysis of independent predictors of mortality in group II: allogeneic.

\begin{tabular}{|l|c|c|c|c|c|}
\multicolumn{1}{c|}{ Predictors } & \multirow{2}{*}{$\mathbf{B}$} & \multirow{2}{*}{ p-value } & Adjusted & \multicolumn{2}{c|}{ O5\% Confidence Interval } \\
\cline { 5 - 7 } & & & Lower & Upper \\
\hline Performance Status & -1.819 & $\mathbf{0 . 0 0 3} *$ & 0.162 & 0.049 & 0.535 \\
\hline Disease of diagnosis & 0.256 & $<\mathbf{0 . 0 0 1} * *$ & 1.291 & 1.121 & 1.487 \\
\hline No of Chemotherapy Cycles & 1.330 & $\mathbf{0 . 0 4 2} *$ & 3.792 & 1.049 & 5.711 \\
\hline Post HSCT: Relapse & 1.596 & $\mathbf{0 . 0 4 4} *$ & 4.934 & 1.079 & 7.567 \\
\hline
\end{tabular}

The logistic regression was performed to ascertain the predictors of in-hospital mortality. The logistic regression model was statistically significant, $\mathrm{p}<0.05$. Patients with performance status, disease of diagnosis, No of chemotherapy cycles and post HSCT: relapse, were the independent predictors of mortality as shown in the table VI-10. 


\section{DISCUSSION}

In our study, in the group of autologous transplantation, there was a statistically significance of overall survival and post HSCT mortality with previous radiotherapy within the group with autologous HSCT with ( $\mathrm{p}$ value was 0.002 ).

Milgrom et al. ${ }^{(5)}$ studied a multi-institutional analysis of peritransplantation radiotherapy in patients with relapsed/refractory Hodgkin lymphoma undergoing autologous stem cell transplantation, they studied 189 patients from 2006 through 2015 who underwent HDC/ASCT and they used radiotherapy within 4 months of ASCT but they found that RT was not found to be associated with LC, progression-free survival, or overall survival on univariate analysis. But in a model incorporating primary refractory HL and FDG-avid disease at the time of HDC/ASCT, RT was found to be associated with a decreased risk of local disease recurrence (hazard ratio, 0.3; $\mathrm{P}=.02$ ). In patients with primary refractory $\mathrm{HL}$ and/or FDG-avid disease at the time of HDC/ASCT, the 4-year LC rate was $81 \%$ with RT versus $49 \%$ without $\mathrm{RT}(\mathrm{P}=.03)$. There was one case of Common Terminology Criteria for Adverse Events grade $\geq 3$ RT-related toxicity (acute grade 3 pancytopenia) ${ }^{(5)}$.

In our study, in the group of allogenic transplantation, we studied a total of 61patients underwent allogenic-HSCT with a total mortality of 29 patients; 16 of them were males and 13 of them were females. Zahid et $\boldsymbol{a l} .{ }^{\left({ }^{(}\right)}$, studied the outcome of allogeneic hematopoietic stem cell transplantation in patients with hematological malignancies. A total of 41 allogeneic transplants were performed for hematological malignancies from April 2004 to December 2012. There were 31 males and 10 females. Median age \pm SD was $28 \pm 11.7$ years (range $8-$ 54 years). Transplant related mortality was $19.5 \%$. The median overall survival was 53.6 months. Overall survival at a median follow up of 37 months was $67 \%{ }^{(\boldsymbol{)})}$.

In our study, in the group of allogenic transplantation, there was a statistically significance at overall survival with performance status $(\mathrm{P}$ value was 0.027) regarding post HSCT relapse and post HSCT mortality. Also in agreement with our results, Sorror $\boldsymbol{e t}$ al. ${ }^{(7)}$ developed the HCT-CI score using a patient cohort of 1055 patients 708 included in the training set and 347 in the validation set. The median age and sex distribution of our group are comparable to their validation set. HCTCI was found to predict outcome for both OS and NRM, thereby representing a helpful instrument in patient counselling. The HCT-CI was developed on a patient cohort observed over a 2-year period. Observed the impact of pretransplant comorbidities persisting even after 65 months, further highlighting the importance of comorbidities on outcome ${ }^{(7)}$.

In our study, in the group of allogenic transplantation, there was a statistically significance at overall survival with No of chemotherapy cycles with $\mathrm{P}=$ 0.028 regarding post HSCT relapse and post HSCT mortality. 11 patients died (37.9\%) in the group that received $\leq 4$ cycles of chemotherapy and 16 patients died $(55.2 \%)$ in the group that received $>4$ cycles of chemotherapy.

Also in agreement with our results Chen $\boldsymbol{e t}$ al. (8) studied 220 consecutive adults with AML in first morphological remission who underwent transplantation after myeloablative or nonmyeloablative conditioning to investigate how the number of standard- or high dose induction courses required to achieve remission impacted post-HCT outcome. Three-year estimates of overall survival were $65 \%$ (95\% confidence interval $[\mathrm{CI}] 56 \%$ to $73 \%$ ), $56 \%$ (95\% CI, $43 \%$ to $67 \%$ ), and $23 \%$ (95\% CI, $6 \%$ to $46 \%$ ) for patients requiring 1 course, 2 courses, or $>2$ courses of induction therapy; corresponding relapse estimates were $24 \%$ (95\% CI, $17 \%$ to $31 \%$ ), $43 \%$ (95\% CI, $31 \%$ to $55 \%$ ), and $58 \%$ (95\% CI, $30 \%$ to $78 \%$ ), respectively. After covariate adjustment (minimal residual disease status, conditioning, age, cytogenetic disease risk, type of consolidation chemotherapy, pre-HCT karyotype, and pre-HCT peripheral blood count recovery), the hazard ratios for 2 or $>2$ induction courses versus 1 induction were 1.16 (95\% CI, .73 to $1.85, \mathrm{P} 1 / 4.53)$ and $2.63(95 \%$ CI, 1.24 to $5.57, \mathrm{P} 1 / 4.011)$ for overall mortality, and 2.10 (95\% CI, 1.27 to 3.48, P $1 / 4.004$ ) and 3.32 (95\% CI, 1.42 to $7.78, \mathrm{P} 1 / 4.006$ ), respectively, for relapse. These findings indicate that the number of induction courses required to achieve morphological remission in AML adds prognostic information for post-HCT outcome that is independent of other prognostic factors ${ }^{(8)}$, and this result was in agreement with results of our study.

In our study, in the group of allogenic transplantation, there was a statistically significance at overall survival with disease of diagnosis with $\mathrm{P}$ value was 0.018 regarding post HSCT relapse and post HSCT mortality. The mortality was $51.1 \%$ of patients diagnosed as AML (15 patients) of total mortality in this group and $6.9 \%$ mortality (2 patients) was diagnosed as CML.

In Indian survey Ganapule et al. (9) had a retrospective descriptive study of all patients with AML who underwent allo-SCT from 1994 to 2013 at their centre to evaluate the clinical outcomes and costeffectiveness of this therapeutic modality. Two hundred fifty-four consecutive patients, median age 34 years, who underwent allo-SCT were included in this study. There were 161 males $(63.4 \%)$. The 5 -year overall survival (OS) and event-free survival for the entire cohort was $40.163 .5 \%$ and $38.763 .4 \%$, respectively. The 5-year OS for patients in first (CR1), second, and third complete remission and with disease/refractory AML was 53.16 5.2\%, $48.268 .3 \%, 31.2617 .8 \%$, and $16.064 .4 \%$, respectively $(\mathrm{P}<0.001)$. From 2007 , reduced intensity conditioning (RIC) with fludarabine and melphalan $(\mathrm{Flu} / \mathrm{Mel})$ was used in a majority of patients in CR1 $(\mathrm{n}=$ 67). Clinical outcomes were compared with historical conventional myeloablative conditioning regimens $(\mathrm{n}=$ 38). Use of Flu/Mel was associated with lower treatmentrelated mortality at 1 year, higher incidence of chronic graft-versus-host-disease, and comparable relapse rates. 
The 5-year OS and event free survival for Flu/Mel and myeloablative conditioning group was $67.266 .6 \%$ versus $38.168 .1 \%(\mathrm{P}=0.003)$ and $63.866 .4 \%$ versus 32.36 $7.9 \%(\mathrm{P}=0.002)$, respectively. Preliminary cost analysis suggests that in our medical cost payment system, RIC allo-SCT in CR1 was likely the most cost-effective strategy in the management of AML and they concluded that in a resource-constrained environment, Flu/Mel RIC allo-SCT for AML CR1 is likely the most efficacious and cost-effective approach in a subset of newly diagnosed young adult patients ${ }^{(9)}$.

John et al. ${ }^{(10)}$ studied long-term survival and late deaths after allogeneic hematopoietic cell transplantation, records of 10,632 patients worldwide reported to the Center for International Blood and Marrow Transplant Research who were alive and disease free 2 years after receiving a myeloablative allogeneic HCT before 2004 for acute myelogenous or lymphoblastic leukemia, myelodysplastic syndrome, lymphoma, or severe aplastic anemia were reviewed. Median follow-up was 9 years, and 3,788 patients had been observed for 10 or more years. The probability of being alive 10 years after HCT was $85 \%$. The chief risk factors for late death included older age and chronic graft-versus-host disease (GVHD). For patients who underwent transplantation for malignancy, relapse was the most common cause of death. The greatest risk factor for late relapse was advanced disease at transplantation. Principal risk factors for non-relapse deaths were older age and GVHD. When compared with age, sex, and nationality-matched general population, late deaths remained higher than expected for each disease, with the possible exception of lymphoma, although the relative risk generally receded over time. They conclude that the prospect for long-term survival is excellent for 2-year survivors of allogeneic HCT. However, life expectancy remains lower than expected. Performance of HCT earlier in the course of disease, control of GVHD, enhancement of immune reconstitution, less toxic regimens, and prevention and early treatment of late complications are needed.

\section{CONCLUSION}

In conclusion, stem cell transplantation has seen a wide array of changes since its implementation in Basel. The patient group eligible for autologous and allogeneic HSCT has diversified towards more advanced patient ages and disease stages and to patients without a family donor, and this is reflected in a higher number of transplants performed over the years. Fiveyear overall and progression-free survival rose for allogeneic and autologous HSCT after the decade of their introduction. After adjustment for factors such as donor type and disease stage, survival improved in every decade. Further efforts in developing regimens that can accommodate the adverse circumstances of higher age, comorbidities and non-availability of a sibling donor, while still providing better control over more advanced diseases, are warranted. To this end, specific strategies are being applied at the time of transplant, such as reduction in regimen toxicity, elaboration of graft-versus-host- disease prophylaxis and better prevention of post-transplant relapse.

\section{RECOMMENDATIONS}

- Using newer prognostic guides to improve patient selection by identifying and treating malignancies at high risk for recurrence.

- Improving the outcome by excluding high-risk patients or those unlikely to benefit from transplantation

- Further highlighting the importance of comorbidities on outcome.

- Minimizing possible errors in scoring the patients.

- Trying to design studies to be prospective data collection.

\section{REFERENCES}

1. Al-toma A, Nijeboer P, Bouma G et al. (2014): Hematopoietic stem cell transplantation for non-malignant gastrointestinal diseases. World J Gastroenterol., 20(46):17368-75.

2. Flomenberg $\mathrm{N}$, Baxter-Lowe LA, Confer $\mathrm{D}$ et al. (2004): Impact of HLA class I and class II highresolution matching on outcomes of unrelated donor bone marrow transplantation: HLAC mismatching is associated with a strong adverse effect on transplantation outcome. Blood, 104: 1923-1930.

3. Campbell S, Sun CL, Kurian S (2009): Predictors of avascular necrosis of bone in long-term survivors of hematopoietic cell transplantation. Cancer, 115: 41274135 .

4. Kaeda J, O'shea D, Szydlo RM et al. (2006): Serial measurement of BCR-ABL transcripts in the peripheral blood after allogeneic stem cell transplantation for chronic myeloid leukemia: an attempt to define patients who may not require further therapy. Blood, 107: 4171-4176.

5. Milgrom SA, Shekeab J (2017): A MultiInstitutional Analysis of Peritransplantation Radiotherapy in Patients With Relapsed/Refractory Hodgkin Lymphoma Undergoing Autologous Stem Cell Transplantation. Cancer, 123(8): 1363-1371.

6. Zahid MF, Ali N, Shaikh MU et al. (2014): Outcome of Allogeneic Hematopoietic Stem Cell Transplantation in Patients with Hematological Malignancies. IJHOSCR., 8: 30-38.

7. Sorror ML, Maris MB, Storb $R$ et al. (2005): Hematopoietic celltransplantation (HCT)-specific comorbidity index: a new tool for risk assessment before allogeneic HCT. Blood, 106(8): 2912-2919.

8. Chen X, Xie H, Wood BL et al. (2015): Relation of clinical response and minimal residual disease and their prognostic impact on outcome in acute myeloid leukemia. J Clin Oncol., 33(11):1258-64.

9. Ganapule A, Sandeep N, Anu K et al. (2017): Allogeneic Stem Cell Transplant for Acute Myeloid Leukemia: Evolution of an Effective Strategy in India. J Glob Oncol., 3(6): 773-781.

10.John R, Navneet SM, Ruta B et al. (2011): Long-Term Survival and Late Deaths After AllogeneicHematopoietic Cell Transplantation. J Clin Oncol., 29:2230-2239. 